\title{
Synthesis of spectrum-compatible bi-directional seismic accelerograms with target elliptical component of polarization
}

\author{
A. Igarashi ${ }^{1} \&$ S. Gigyu ${ }^{2}$ \\ ${ }^{I}$ Disaster Prevention Research Institute, Kyoto University, Japan \\ ${ }^{2}$ Department of Urban Management, \\ Graduate School of Engineering, Kyoto University, Japan
}

\begin{abstract}
Unidirectional spectrum-matched accelerograms are generally adopted in typical design specifications to assess the seismic performance of structures. Considering that actual earthquake ground motions are of bi-directional nature in horizontal components, use of spectrum-matched bi-directional accelerograms should be advantageous to reliable seismic performance assessment of curved bridges or brides with energy dissipation device and seismic isolators. In this study, a procedure to synthesize spectrum-matched bi-directional accelerograms is proposed. The idea of the proposed procedure is to combine a conventional spectrum-matched unidirectional accelerogram and its Hilbert transform as the two orthogonal components of the bi-directional accelerogram, so that the elliptical component of polarization calculated by Vidale's complex polarization analysis, for the resulting bi-directional accelerogram is matched to the target value with a simple scaling. Moreover, the response of a bi-directional nonlinear elasto-plastic single-mass system model to synthesized bi-directional ground motions is investigated. It is found that the influence of the elliptical component of polarization on the nonlinear response depends on the frequency content and phase characteristics of the accelerogram as well as the natural period of the structure. A suitable choice of the parameter expressing the elliptical component is recommended for relevant seismic performance assessment.
\end{abstract}

Keywords: bi-directional excitation, bi-directional response, seismic performance assessment, Hilbert transform, complex polarization filter, nonlinear response. 


\section{Introduction}

Earthquake ground motion is of three-dimensional nature consisting of a bidirectional motion in the horizontal components and unidirectional one in the vertical component. Therefore, the seismic response of structures is threedimensional and the horizontal components of the ground motion are especially important for the assessment of structures, namely the bridges. However, in typical seismic design codes for highway bridges, in Japan [1] for example, seismic performance assessment is conducted by nonlinear time history analysis using unidirectional spectrum-matched accelerograms to excite in the longitudinal direction and in the transverse direction separately. This is because reliable bi-directional restoring force models of structural members applicable to the seismic performance assessment in design and construction practice are still on the developing stage. However, owing to research efforts by engineers and researchers in the development of modelling and assessing the bi-directional behaviour of structures, numerical analysis of bi-directional structural response can be used if appropriate structural modelling is achieved.

In order to investigate the bi-directional seismic performance of structures, one of the viable options is to use ground motion accelerograms recorded during past earthquakes. However, the accelerograms of actual earthquakes do not match with the design spectrum to be used in seismic performance assessment. Although several methods to synthesize spectrum-matched bi-directional ground motions have been investigated in past research, the practical procedure of synthesis is not yet established. For example, Grant [2] developed a method to generate two horizontal ground motion components matched to the target response spectra with the use of weighted wavelets for spectral adjustment. Igarashi et al. [3] proposed a method for synthesis of bi-directional accelerograms consisting of a spectrum-matched uni-directional accelerogram and its Hilbert transform, referred to as the complementary waves, as the orthogonal components. Nevertheless, some problems remain in the latter approach, including the slightly conservative result of the obtained seismic response compared with the conventional uni-directional excitation case, and omission of detailed multi-dimensional characteristics of the real earthquakes.

In this study, a procedure to synthesize spectrum-matched bi-directional accelerograms with specified values of the elliptical component of polarization applicable to seismic performance assessment is proposed. To discuss the implication of the accelerograms synthesized with the proposed method in the seismic performance assessment, influence of the elliptical component of polarization on the dynamic response of nonlinear elasto-plastic structures is investigated by numerical dynamic analysis of an idealized simple dynamic model.

\section{Analysis of bi-directional accelerograms}

As the preliminary of proposed method, the fundamental concepts to analyze the nature of bi-directional ground motion are described in this section. 


\subsection{Bi-axial response spectrum}

Isotropic elastic single-mass models with various natural periods $T$ and a damping ratio $h=0.05$ are assumed to be subjected to bi-directional ground motion (as shown in Fig. 1). The bi-directional dynamic response of the singlemass system is computed by dynamic analysis using a numerical step-by-step time integration scheme. When the two components of the bi-directional structural response are plotted on the horizontal plane as a trajectory, relationship between the peak distance of the response acceleration trajectory from the origin and the natural period $T$ of the structural model is referred to as the bi-axial response spectrum [4]. The concept of bi-axial response spectrum is an extension of the elastic response spectrum to the two-dimensional case. When the bi-axial response spectrum of the bi-directional ground motion is compatible with the target elastic response spectrum, the said bi-directional input ground motion is defined to be spectrum-matched in this study.

\subsection{Elliptical component of polarization}

Vidale [5] proposed the complex polarization filter to characterize the threedimensional particle motion trajectory associated with earthquake ground motion due to the seismic wave propagation. In the application of the complex polarization filter, an indicator for the ellipticity of the trajectory is called the elliptical component of polarization, denoted by the symbol $P_{E}$. The elliptical component of polarization is computed with the following procedure. Let $a_{x}(t)$ and $a_{y}(t)$ be the components of the ground motion in the $x$ and $y$ directions at time $t$.

1. Determine the analytic signals $u(t)$ and $v(t)$ defined by Eq. (1).

$$
\begin{aligned}
& u(t)=a_{x}(t)+i H\left[a_{x}(t)\right] \\
& v(t)=a_{y}(t)+i H\left[a_{y}(t)\right]
\end{aligned}
$$

where $i$ is the imaginary unit, $H[]$ represents the Hilbert transform expressed by

$$
H[x(t)]=\frac{1}{\pi} \int_{-\infty}^{\infty} \frac{x(u)}{t-u} d u
$$

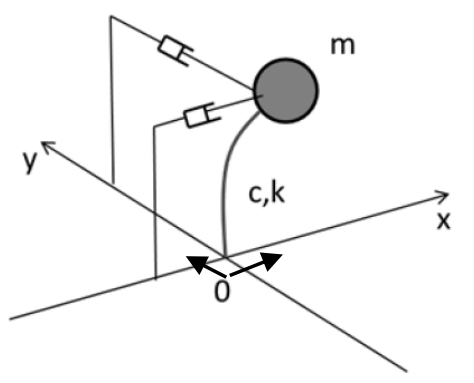

Figure 1: Bi-directional single-mass model. 
2. At each time $t$, compute the complex covariance matrix $\mathbf{C}(t)$.

$$
\mathbf{C}(t)=\left[\begin{array}{ll}
u u^{*} & u v^{*} \\
v u^{*} & v v^{*}
\end{array}\right]_{t}
$$

where the superscript '*' represents complex conjugate.

3. The matrix $\mathbf{C}(t)$ is Hermitian and its eigenvalues are nonnegative real values. Find the eigenvector $\chi_{1}$ corresponding to the largest eigenvalue. The eigenvector $\chi_{1}$ is assumed to be normalized so that its norm equals to unity.

$$
\chi_{1}(t)=\left\{\begin{array}{l}
x(t) \\
y(t)
\end{array}\right\}
$$

4. Find $X(t)$ by

$$
X(t)=\max _{\alpha} \sqrt{\left\{\operatorname{Re}\left(x(t) e^{i \alpha}\right)\right\}^{2}+\left\{\operatorname{Re}\left(y(t) e^{i \alpha}\right)\right\}^{2}}
$$

5. The elliptical component of polarization $P_{E}(t)$ is computed by

$$
P_{E}(t)=\frac{\sqrt{1-X(t)^{2}}}{X(t)}
$$

The value of $P_{E}$ is computed in the range between zero and unity. A linearly polarized motion and a circularly polarized motion correspond to $P_{E}=0$ and $P_{E}=1$, respectively (as shown in Fig. 2).

\section{Synthesis of spectrum matched bi-directional ground motion with given $P_{E}$}

The bi-directional accelerogram that matches with a specified response spectrum $S_{a}(T)$ and a specified value of the elliptical component of polarization $\alpha(0 \leqq \alpha \leqq$ 1) can be synthesized by the following procedure.

1. Prepare a unidirectional accelerogram $a_{x}(t)$ matching the given spectrum $S_{a}(T)$. In the Japanese highway bridge design standard, for example, standard accelerograms satisfying this condition are provided.

2. Compute the Hilbert transform of $a_{x}(t)$ and denote the result by $a_{y}(t)$.

$$
a_{y}(t)=H\left[a_{x}(t)\right]
$$

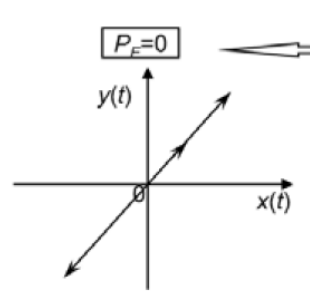

linear

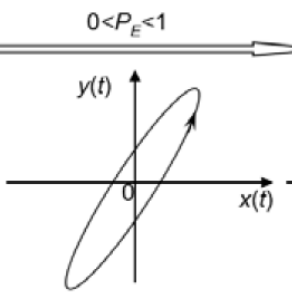

elliptic

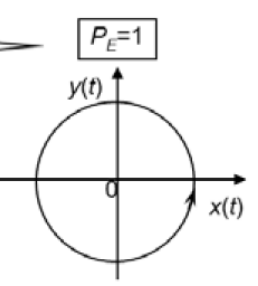

circular

Figure 2: Concept of elliptical component of polarization. 
3. Define the direction angle $\theta$ with respect to the $x$ axis in an orthogonal $x-y$ coordinate system. By scaling the trajectory of the vector $\left\{a_{x}(t), a_{y}(t)\right\}$ with $\alpha$ in the directional orthogonal to $\theta$, compute the vector $\left\{a_{x}{ }^{\prime}(t), a_{y}{ }^{\prime}(t)\right\}$ using the following expression.

$$
\left\{\begin{array}{l}
a_{x}{ }^{\prime}(t) \\
a_{y}{ }^{\prime}(t)
\end{array}\right\}=\left[\begin{array}{cc}
\cos \theta & -\sin \theta \\
\sin \theta & \cos \theta
\end{array}\right]\left[\begin{array}{cc}
\cos \theta & \sin \theta \\
-\alpha \sin \theta & \alpha \cos \theta
\end{array}\right]\left\{\begin{array}{l}
a_{x}(t) \\
a_{y}(t)
\end{array}\right\}
$$

The vector $\left\{a_{x}{ }^{\prime}(t), a_{y}{ }^{\prime}(t)\right\}$ is the desired bi-directional input.

The value of $\alpha$ can be determined based on the character of actual seismic records. For example, the trajectory of the ground motion recorded at Higashi Kobe Bridge during the 1995 Kobe Earthquake is shown in Fig. 3(a). The relationship between the absolute values of the acceleration $\left(a_{E W^{2}}(t)+a_{N S}{ }^{2}(t)\right)^{1 / 2}$ and $P_{E}(t)$ for this record is shown in Fig. 3(b). The weighted average of $P_{E}$ is found to be 0.257 , which can be specified as the value of $\alpha$. The accelerogram of the bi-directional input synthesized with the above procedure using a standard wave for Level-2, Type II earthquake for Type III soil condition II-III-3, $\theta=50^{\circ}$

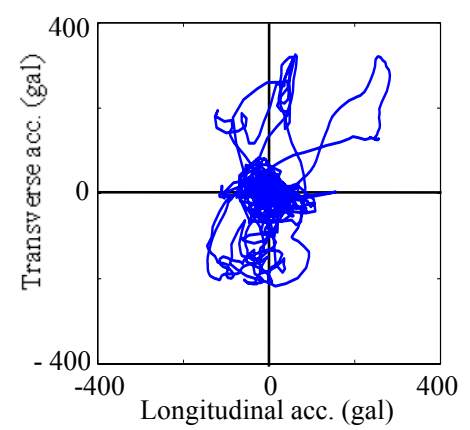

(a) Trajectory of horizontal components

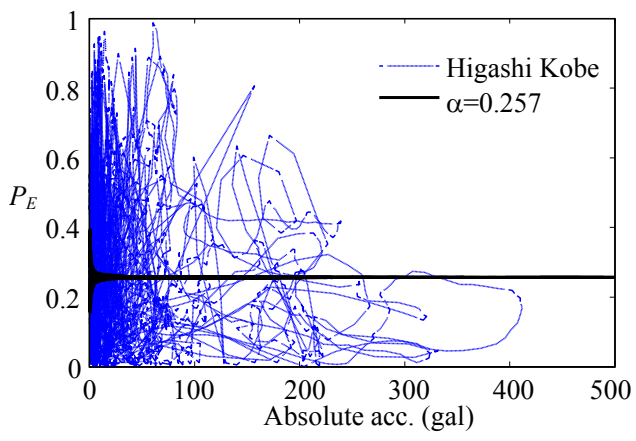

(b) Absolute value of acceleration $-P_{E}$ plot

Figure 3: 1995 Kobe earthquake, Higashi Kobe Bridge record.
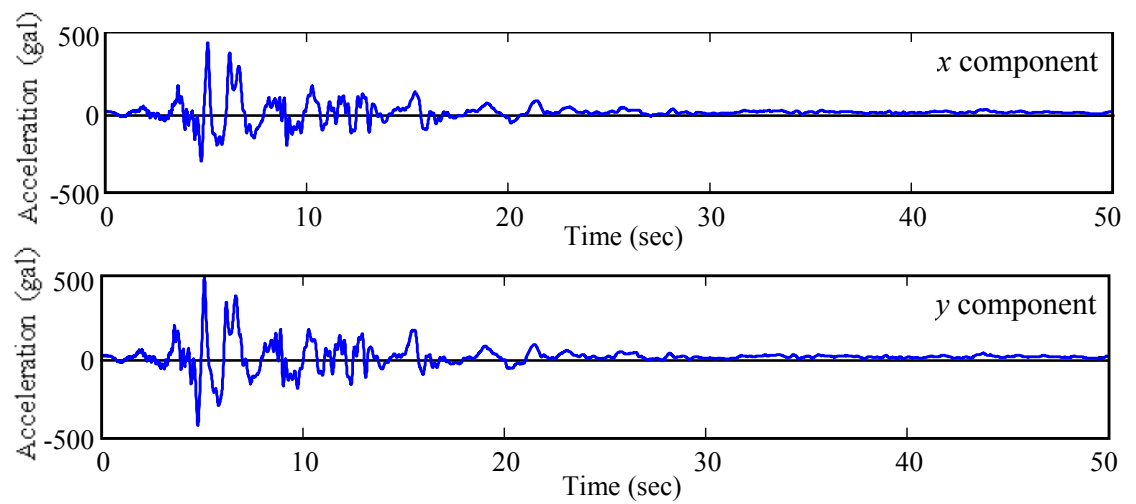

Figure 4: Synthesized bi-directional accelerogram. 
and $\alpha=0.257$ is shown in Fig. 4 . The trajectory of the synthesized bi-directional accelerogram is shown in Fig. 5. As indicated by the relationship between the absolute acceleration and $P_{E}$ for the synthesized accelerogram shown in Fig. 3(b), the overall shape of the trajectory is an ellipse with an aspect ratio of the specified $\alpha$. The bi-axial spectra of the synthesized bi-directional ground motion using various values of $\alpha$ ranging from zero to unity, with the same standard wave and $\theta$, are shown in Fig. 6 . As indicated by the figure, the bi-axial response spectra show a good agreement with the target spectrum with sufficient accuracy.

\section{Nonlinear structural response to synthesized bi-directional ground motion}

The effect of $P_{E}$ of the bi-directional input on the bi-directional response of nonlinear elasto-plastic structural system models, is investigated by numerical dynamic analysis. The Multi-Shear-Spring (MSS) model shown in Fig. 7 is used

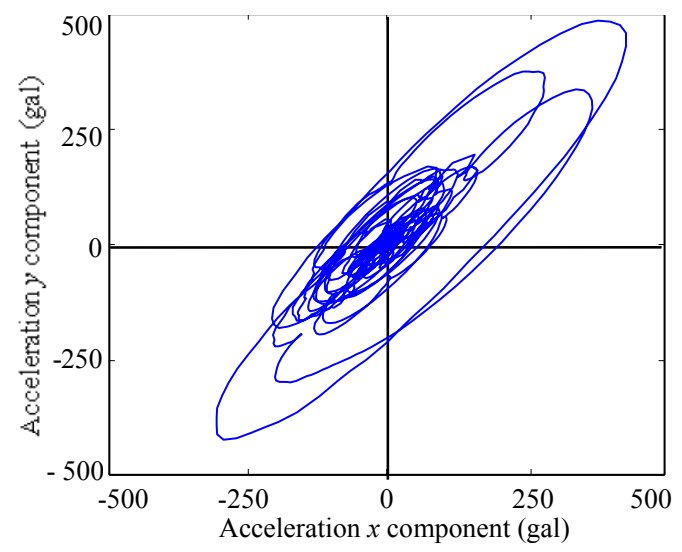

Figure 5: Trajectory of horizontal components of synthesis result $(\alpha=0.257)$.

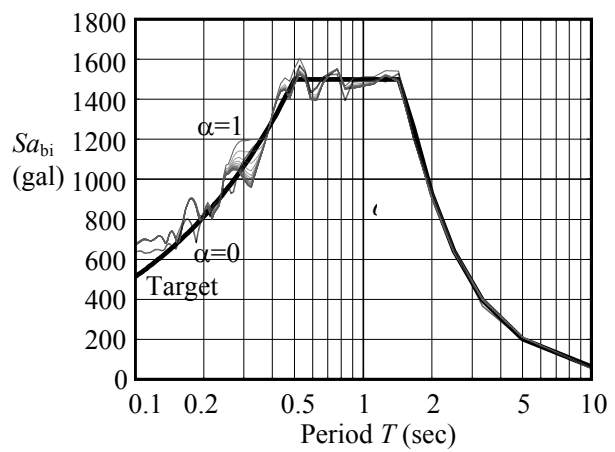

Figure 6: Bi-axial response spectra for various values of $\alpha$. 


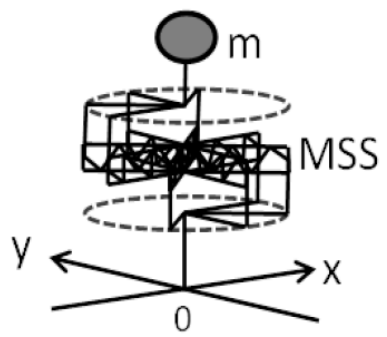

Figure 7: $\quad$ Single mass system with multi shear spring (MSS) model.

Table 1: $\quad$ Parameters and cases.

\begin{tabular}{|c|c|c|c|}
\hline Mass $m$ & \multicolumn{3}{|c|}{$50(\mathrm{t})$} \\
\hline Damping ratio $h$ & \multicolumn{3}{|c|}{0.05} \\
\hline $\begin{array}{c}\text { Secondary } \\
\text { stiffness ratio }\end{array}$ & Case-1 & Case-2 & Case-3 \\
\hline & $0.4(\mathrm{~s})$ & $1.0(\mathrm{~s})$ & $1.8(\mathrm{~s})$ \\
\hline Natural period $T$ & $12337\left(\mathrm{t} / \mathrm{s}^{2}\right)$ & $1974\left(\mathrm{t} / \mathrm{s}^{2}\right)$ & $609\left(\mathrm{t} / \mathrm{s}^{2}\right)$ \\
\hline Initial stiffness & $12.9(\mathrm{~mm})$ & $124.2(\mathrm{~mm})$ & $402.5(\mathrm{~mm})$ \\
\hline Yield displ. & 19.9 & &
\end{tabular}

Table 2: $\quad$ Parameters of bi-directional accelerogram synthesis.

\begin{tabular}{|c|c|}
\hline Base accelerogram $a_{x}$ & JRA Standard Wave II-III-3 \\
\hline Elliptical component $\alpha$ & $0 \leq \alpha \leq 1$ \\
\hline Direction angle $\theta$ & 50 degree \\
\hline
\end{tabular}

to represent the bi-directional elasto-plastic behaviour of structures, and the model parameters are summarized in Table 1. Three initial natural periods are selected in the short, medium and long period ranges, which are referred to as Case-1, Case-2 and Case-3, respectively. The parameters used to synthesize the input waves are shown in Table 2 .

The displacement response trajectory and the restoring force characteristics in Case 1 are shown in Figs 8 and 9, respectively. In Fig. 8, the radius of the circle of dotted line represents the peak displacement response computed by the conventional unidirectional standard wave, and the displacement response trajectory computed for the bi-directional input of $\alpha=1$, corresponding to the standard and complementary waves, is also plotted with a thin line.

The relationship between the peak displacement response and the level of elliptical component is shown in Fig. 10. Three solid lines show the results computed by the bi-directional inputs synthesized with various $\alpha$ in the range between zero and unity in each case, and the vertical line indicate the location of 


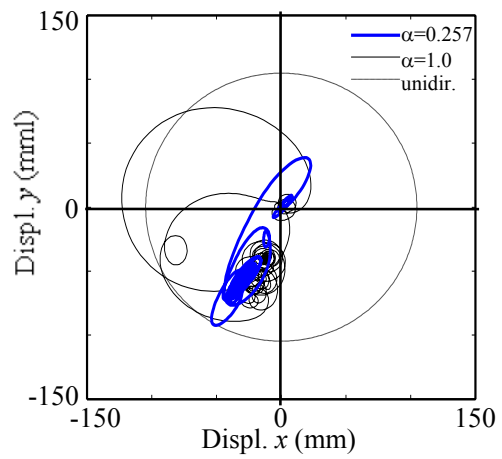

Figure 8: Response displacement trajectory.

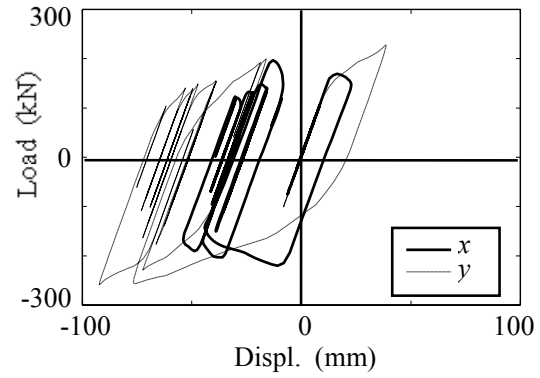

Figure 9: Restoring force response.

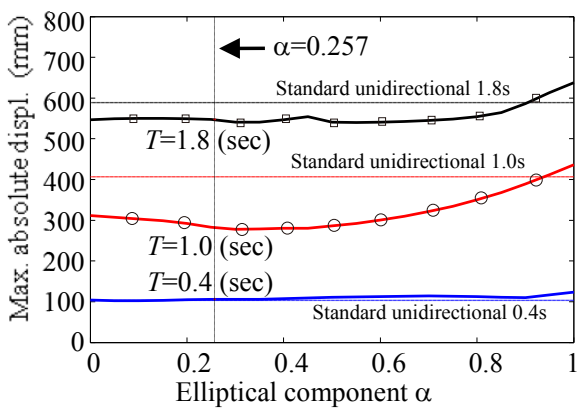

Figure 10: Influence of elliptical component of polarization on structural response.

$\alpha=0.257$ in this plot. The dashed lines indicate the structural response for the unidirectional standard wave case. As shown in Fig. 10, the change of peak displacement is neither monotonic increase nor decrease, and the effect of the elliptical component is also influenced by the natural period of the structure. 
Compared with the dynamic response to the conventional unidirectional standard accelerograms, the analysis using the proposed bi-directional inputs does not necessarily result in a greater response, although the response for the case of $\alpha=1$ tends to be larger than the unidirectional input. Results of the numerical analysis using other standard waves given in the Japanese seismic design standard indicate a similar tendency in the variation of the effect of the elliptical component, depending on the selection of the base unidirectional spectrummatched accelerogram. Although it can be claimed that selecting the parameter for the elliptical component of polarization $\alpha=1$ provides conservative assessment, A suitable choice of the parameter $\alpha$ is recommended for relevant seismic performance assessment.

\section{Conclusions}

In this study, a procedure to synthesize spectrum-matched bi-directional accelerograms with the specified elliptical component of polarization is proposed. In order to examine the character of the accelerograms synthesized by this procedure and significance in the seismic performance assessment of structures, the response of a bi-directional nonlinear elasto-plastic system models to the synthesized accelerograms is investigated. It is found that the influence of the elliptical component of polarization on the nonlinear structural response is not monotonic, and it depends on the frequency content and the phase characteristics of the accelerogram as well as the natural period of the structures. A suitable choice of the parameter expressing the elliptical component is recommended for relevant seismic performance assessment.

\section{Acknowledgement}

The research described here is financially supported by the Japan Society of Promotion of Science, Grant-in-Aid for Scientific Research (B) (Grant No. 25289136).

\section{References}

[1] Japan Road Association, Specification for highway bridges, Part V Seismic Design, 2012 (in Japanese).

[2] Grant, D.N., Response spectral matching of two horizontal ground-motion components, ASCE Journal of Structural Engineering, Vol. 137, No. 3, pp. 289-297, 2011.

[3] Igarashi, A., Inoue, K., Furukawa, A., Uno, H. \& Matsuda, H. Synthesis of bi-directional seismic ground motion by standard and complementary waves for seismic design of bridges, Journal of Japan Society of Civil Engineers, Ser. A1 (SE/EE), Vol. 68, No. 4, pp. I_458-I_469, 2012 (in Japanese). 
72 Earthquake Resistant Engineering Structures X

[4] Gavin, H. P. \& Dickinson, B. W., Generation of uniform-hazard earthquake ground motions, ASCE Journal of Structural Engineering, Vol. 137, No. 3, pp. 423-432, 2011.

[5] Vidale, J.E., Complex polarization analysis of particle motion, Bull. Seism. Soc. Am., Vol. 76, No. 5, pp. 1393-1405, 1986. 